\title{
Toxic effects of mineral oil saturated hydrocarbons (MOSH) and relation to accumulation in rat liver
}

Unni Cecilie Nygaard ${ }^{a *}$, Åshild Vege ${ }^{b, c}$, Torleiv Rognum ${ }^{b, c}$, Koni Grob ${ }^{\mathrm{d}}$, Christel Cartier ${ }^{\mathrm{e}}$, Jean-Pierre Cravedie, Jan Alexander ${ }^{\mathrm{a}}$

${ }^{a}$ Division of Infection Control and Environmental Health, Norwegian Institute of Public Health, P.O.Box 4404 Nydalen, N-0403 Oslo, Norway.Unni.cecilie.nygaard@fhi.no.jan.alexander@fhi.no

${ }^{b}$ Department of Forensic Medicine, University of Oslo, Norway

'Department for Forensic Sciences, Oslo University Hospital, Norway. aasveg@ous-hf.no trognum@ous-hf.no

${ }^{d}$ Official Food Control Authority of the Canton of Zurich, Fehrenstrasse 15, P.O.Box, CH-8032 Zurich, Switzerland. koni@grob.org

${ }^{\text {e}}$ Toxalim (Research Centre in Food Toxicology), INRA, ENVT, INP-Purpan, UPS, Toulouse, France. jeanpierre.cravedi@inra.fr; christel.cartier@inra.fr

\footnotetext{
* Corresponding author: Unni Cecilie Nygaard, Department of toxicology and risk assessment, Norwegian Institute of Public Health, P.O.Box 4404 Nydalen, N-0403 Oslo, Norway. Phone: +472107 6435. E-mail: Unni.cecilie.nygaard@fhi.no
} 


\section{Abstract}

Humans are daily exposed to mineral oil saturated hydrocarbons (MOSH) from the diet. We exposed female Fischer 344 rats to a broad mixture and sub-fractions of MOSH. Chemical characterization of the MOSH mixture used and material accumulated in rat tissues were previously reported (Barp et al 2017a, b). Rats were exposed to feed containing 0-4000 $\mathrm{mg} / \mathrm{kg}$ broad MOSH mixture for 30, 60, 90 and 120 days; and for 120 days to feed containing different MOSH fractions: i) mainly molecular masses <C25 (S-C25), ii) dewaxed, mainly molecular masses >C25 (L-C25) and iii) the L-C25 fraction mixed with wax largely consisting of $n$-alkanes $>$ C25 (L-C25W). Treatments related effects were increased liver and spleen weight, as well as vacuolization and granuloma formation with lymphoid cell clusters in the liver, but effects varied strongly between the MOSH fractions tested. We conclude that increased liver and spleen weights were mainly related to accumulated iso-alkanes and substituted cycloalkanes, but also wax n-alkanes. Induction of liver granuloma appeared to be related to n-alkanes $>\mathrm{C} 25$ and not to the accumulated amount of $\mathrm{MOSH}$. Immune responses to an injected antigen were not affected. MOSH fractions associated with increased liver and spleen weights were similar to those accumulating in humans.

Keywords: mineral oil, mineral wax, n-alkane, rat, granuloma, immune function 


\section{Introduction}

Mineral oil hydrocarbons $(\mathrm{MOH})$ are complex mixtures of mineral oil aromatic hydrocarbons (MOAH) and mineral oil saturated hydrocarbons (MOSH). MOSH includes paraffins (consisting of linear and branched alkanes) and naphthenes (consisting of mainly alkylated cycloalkanes). Their wide use for various applications causes $\mathrm{MOH}$ to enter the food chain from many sources, following intended use as food additives and in packaging material, or as contaminants such as printing ink in recycled paper (EFSA, 2012). $\mathrm{MOH}$ composition varies depending on the crude oil source and treatment, thus humans are exposed to a broad range of $\mathrm{MOH}$ of complex and mostly unknown composition.

A number of toxicity studies carried out in rodents with different food-grade $\mathrm{MOH}$ (essentially MOSH) have indicated that repeated exposure to most of those grades caused tissue bioaccumulations and liver granuloma formation associated with inflammatory responses in rats (EFSA, 2012). The various food-grade MOSH tested, characterized in terms of physio-chemical properties, differed both in their potential for bioaccumulation and granuloma formation. MOSH with carbon numbers in the range between 16 and 40 (C16C40) may accumulate in various tissues in both humans and experimental animals (Barp et al., 2014; EFSA, 2012). The relationship between exposures to different MOSH mixtures characterized in terms of chemical composition and the bioaccumulation, granuloma formation as well as related inflammatory responses and possible effects on immune function is not clear. With the exception of waxes, mainly consisting of n-alkanes, that appear to be a potent inducer of liver granuloma in Fischer 344 rats, the types of hydrocarbons of main concern within the MOSH mixtures has not been possible to identify. 
None of the mixtures hereto tested were representative of the broad range of hydrocarbons to which humans are exposed via the food. In its risk assessment of MOSH, the EFSA CONTAM Panel (EFSA, 2012) recommended that efforts should be made to base the toxicological evaluation of $\mathrm{MOH}$ on molecular mass ranges and structural subclasses, rather than on physico-chemical properties. In addition, the CONTAM Panel recommended further investigation of the possible relationship between oral exposure to MOSH and immunological effects in humans.

In the present study, which is part of a larger study, we used female Fischer 344 rats because they are known to be a sensitive model with regard to both MOSH accumulation and granuloma formation in the liver (Firriolo et al., 1995; Griffis et al., 2010). First, we investigated the effects of a broad MOSH mixture ranging from about $\mathrm{C} 14$ to $\mathrm{C} 50$ and covering the broad human exposure. In a second experiment, we tested three sub-fractions in order to identify the MOSH having the highest toxicological potential. While we in two previous publications provide characterizations of the chemical composition of the mixtures and compositions of MOSH and amounts found in the rat tissues (Barp et al. 2017a,b), the present study provides information on weight gain and liver and spleen weights, liver pathology and effects on immune function assessed as the primary IgM antibody response to an antigen. Our specific aims were to: i) assess the oral liver toxicity of a broad MOSH mixture representative of the whole MOSH range to which humans are exposed via the diet, ii) identify the fraction(s) and sub-categories/chemical structure with the main toxic potential in the liver, iii) investigate the relation between MOSH accumulation in the liver and the formation of hepatic granuloma, and iv) explore the putative changes in immune function assessed as primary antibody production. 


\section{Materials and methods}

\subsection{Animals}

All animal experiments were performed according to the EU Directive 2010/63/EU on the protection of animals used for scientific purposes, and were approved by the French ministry of research and education (APAFIS4303-2016022912425905). Four week old female Fischer 344 rats were entering the experiment after one week of acclimatization. Animals were randomly allocated to the cages, and kept under temperature and light control $\left(21^{\circ} \mathrm{C} \pm 2{ }^{\circ} \mathrm{C}\right.$, $12 \mathrm{~h}$ light/dark cycle). Pelleted feed (based on AIN-93 M commercial diet) and tap water were available ad libitum.

\subsection{Experiment 1 - a broad MOSH mixture}

In the first experiment, groups of animals were exposed to $0,40,400$ and $4000 \mathrm{mg} / \mathrm{kg}$ feed of a broad MOSH mixture for 30, 60, 90 and 120 days. To investigate the possible depuration of accumulated hydrocarbons and possible regression of granuloma formed in the liver, additional groups of rats exposed to 40,400 and $4000 \mathrm{mg} / \mathrm{kg}$ MOSH during 90 days were fed the control diet during the last month (called $90+30 \mathrm{~d}$ in the following). Five animals were randomized into each cage, and four cages were randomly assigned to the control group and the remaining cages randomly assigned to one of the other groups (five cages per group). Feed consumption was recorded biweekly while body weights were recorded weekly. After 30, 60, 90 and 120 days of exposure, five animals per group (from 5 different cages) were sacrificed by $\mathrm{CO}_{2}$ inhalation. The liver, spleen and abdominal adipose tissue were excised, weighed and immediately frozen in liquid nitrogen before storage at $-20^{\circ} \mathrm{C}$ for chemical 
analyses. The exception was the liver left lobes; these were fixed in $4 \%$ neutral buffered formalin before being processed for conventional histopathological examination by mounting in paraffin. The paraffin blocks were shipped to NIPH in Oslo, where they were sectioned and stained (see below).

Heparinized blood samples collected from animals sacrificed after 30, 60 and 90 days were stored at $-20^{\circ} \mathrm{C}$. The gastrointestinal tract (including digestive content) was excised, homogenized and frozen for potential chemical analysis. Liver, adipose tissue, spleen and remaining carcasses were shipped to $\mathrm{KLZH} /$ Zurich for later MOSH tissue analysis, as previously described and reported in Barp et al. (2017a).

As a measure of immune function, the KLH IgM antibody assay was conducted according to the OECD test guideline 443. Briefly, all rats exposed for $120 \mathrm{~d}$ received an injection (i.v.) of an antigen $(300 \mu \mathrm{g} / \mathrm{kg}$ bw native HMW keyhole limpet hemocyanin, $K L H$, from Stellar biotechnologies, CA, USA) 5 days before the end of the experiment. A terminal blood sample from vena cava was collected in glass tubes without anticoagulant, serum was prepared and frozen at $-80^{\circ} \mathrm{C}$ in Eppendorf tubes. The serum samples were sent to NIPH/Oslo on dry ice.

\subsection{Experiment 2 - MOSH sub-fractions}

Female rats were randomly assigned to 20 cages and groups ( 4 animals per cage, 8 animals per group), and exposed to three different MOSH fractions in feed concentrations of 0,400 , 1000 and $4000 \mathrm{mg} \mathrm{MOSH} / \mathrm{kg}$. The experimental conditions were as described for experiment 1 . Weekly body weight and feed consumption were recorded. Animals were euthanized after 120 days of exposure, and liver, spleen and adipose tissue were excised and processed for chemical (Barp et al., 2017b) and histological analyses, as described above. 
The KLH assay was performed in all rats, as described above, with the exception that the rats were subcutaneously injected with $200 \mu \mathrm{L}$ of $5 \mathrm{mg} / \mathrm{mL}$ KLH (PI-77600 from Thermo Scientific, Pierce) in PBS buffer as previously reported (Vandebriel et al., 2014).

\subsection{Preparation of the MOSH mixtures}

As previously described (Barp et al., 2017a), a broad MOSH mixture was prepared for use in experiment 1, ranging from about $\mathrm{C} 14$ to about C50. Briefly, a similar concentration per mass was achieved over a wide mass range by combining the following products: $295 \mathrm{~g} / \mathrm{kg}$ paraffin wax Ph Eur, low viscosity, Fluka 76233 (Buchs, Switzerland), 295 g/kg paraffin wax Ph Eur, high viscosity, Fluka 76234, 147 g/kg Catenex Ph 941 FU (Shell) and 263 g/kg distillate from paraffin highly liquid Ph Eur, BP NF, Merck JP K43210074 2091.17174 .1000

(Darmstadt, Germany). The range of molecular masses was intended to be broader than that previously observed in human tissues (approximately C16-C45; (Barp et al., 2014)).

For experiment 2, three fractions, S-C25, L-C25 and L-C25W, were prepared as previously described (Barp et al., 2017b), using the oils that made up the central part of the broad mixture used in experiment 1. S-C25 (Fluka paraffin wax PhEur, low viscosity, 76233), which made up $32.8 \%$ of the broad mixture consisted mainly of hydrocarbons with masses below C25. L-C25 (Fluka paraffin wax Ph Eur, high viscosity, 76234), which also made up $32.8 \%$ of the broad mixture, consisted of MOSH mainly with masses above C25. L-C25W consisted of a 1:1 (w:w) mixture of L-C25 and a wax (411663 from Sigma-Aldrich) of similar mass range (mp $\geq 65^{\circ} \mathrm{C}$ ). $n$-Alkanes were virtually absent in L-C25, whereas S-C25 contained a minor proportion of wax-hydrocarbons (Barp et al., 2017b). 


\subsection{Feed preparation}

A standard pelleted diet (AIN-93M, Table 2) was used, as described by Reeves and coworkers (Reeves et al., 1993). Background levels of MOSH or polyolefin oligomers (POSH) in the major feed ingredients were found to be low (Barp et al., 2017a). The MOSH mixtures were dissolved in soybean oil and stirred for 4 hours at $40{ }^{\circ} \mathrm{C}$ before being incorporated into the feed.

For experiment 1, the broad MOSH mixture was incorporated into the feed at 40, 400 and $4000 \mathrm{mg} / \mathrm{kg}$ feed (an equivalent mass of soybean oil being replaced by the MOSH), which covered the range from below to above the doses causing liver granuloma in previous experiments (EFSA, 2012). The MOSH concentrations in the feed preparations were within $88-95 \%$ of the nominal concentrations, and the control feed contained $1.6 \mathrm{mg} \mathrm{MOSH} / \mathrm{kg}$ feed (Barp et al. 2017a).

For experiment 2, the three MOSH sub-fractions were added to the feed aiming at 400,1000 and $4000 \mathrm{mg} / \mathrm{kg}$ (Barp et al., 2017b). The lowest concentration $(40 \mathrm{mg} / \mathrm{kg}$ ) in experiment 1 was omitted in experiment 2 , as no granuloma had been formed at the lowest doses. Since the more narrow fractions increased the concentrations in their range, a dose of $1000 \mathrm{mg} / \mathrm{kg}$ was included in this experiment. Feed samples were measured to be within $84-99 \%$ of the nominal concentrations (Barp et al., 2017b).

\subsection{Histopathological analyses of the livers}

Paraffin tissue blocks from left liver lobes (see above) were sectioned at $4 \mu \mathrm{m}$ by use of a microtome. Four sections from each tissue block were stained with hematoxylinazophloxine-saffron (HAS) for morphometric quantification of granuloma, clusters of 
lymphoid cells in portal tracts, clusters of lymphoid cells (separate from the granuloma) in liver parenchyma and semi-quantitative evaluation of the degree of liver cell vacuolization. Not all granuloma were surrounded by lymphocytes, consistent with previous findings (Fleming et al., 1998). Sixty serial sections were cut from each tissue block and the first, the 20th, 40th and 60th section were selected for staining, aiming to avoid counting of the same granuloma and lymphoid cell clusters in the different sections.

All sections were histologically examined and evaluated in a blinded fashion by the same examiner on Leica DMLB microscopes. The reproducibility of histopathological evaluation of granuloma was evaluated and found satisfactory (by applying a k-test; between observers $\mathrm{k}=0.6, \mathrm{p}<0.05$ (moderate), and for the same observer over time $\mathrm{k}=0.87, \mathrm{p}<0.05$, (very good)). Initially, seven sections from two cases were evaluated to determine the number of sections necessary to obtain a representative granuloma density from each liver lobe. Since the variations in granuloma density in each lobe was relatively modest, it was decided to examine four sections from each tissue block. In each section, we counted all granuloma and lymphoid cells clusters in portal tracts and liver parenchyma. Liver cell vacuolization was scored semi-quantitatively as none, slight ( $<5 \%$ of liver cells), moderate (5-15\%) and abundant (> $15 \%$; see also Fig. 4). For graphical presentation and statistical analyses, the scores were denoted 0, 1, 2 and 3, respectively. The granuloma and lymphoid cells clusters are reported as density $\left(\right.$ per $\mathrm{cm}^{2}$ ). A Wild Heerbrugg stereo microscope equipped with a 6 times magnification lens and a photo tube with a reduction ocular (0.5), or a Leica MZ 12 stereo microscope calibrated to give the same magnification, were used to measure the tissue section areas. The picture was transmitted to the planimetry unit by a Leica DFC450 camera, and area calculation was done by a LEICA application system (LAS). 


\subsection{KLH-specific IgM antibody detection}

After thawing of the serum samples, the KLH-specific IgM antibody concentrations were determined in duplicate by an enzyme-linked immunosorbent assay (ELISA; ELI-02M, Stellar biotechnologies, CA, USA), according to the manufacturer's instructions. In experiment 1 , the optimal serum dilution was found to be 1:2000. In experiment 2 , however, this dilution gave KLH-specific IgM levels above the assay detection limit for many samples, thus, the reported results were obtained in 1:5000 dilutions of the sera. The higher serum concentrations of KLH-specific IgM antibodies in experiment 2 may be explained by the change in KLH provider and/or injection route (s.c. versus i.v.; see above).

\subsection{Statistical analyses}

For animal and tissue weights endpoints, one way ANOVA analysis and Dunnett's multiple comparisons test were used. For all other endpoints we chose to use non-parametric ANOVA (Kruskal-Wallis) with Dunn's post hoc test correcting for multiple comparisons, due to the small group sizes $(n=5-8)$ and the non-normal distributions. The relationship between external exposure or MOSH concentrations in liver and granuloma density was examined by fitting an exponential growth curve by a least squares (ordinary) best fit model. This model was chosen based on the apparent exponential pattern of the data sets for granuloma formation as a function of MOSH in feed. The statistical analyses were all performed in GraphPad Prism 7 software. 


\section{Results}

\subsection{Experiment 1 - a broad MOSH mixture}

Three dose levels of a broad MOSH mixture were administered to rats via the feed for 30 , 60,90 and 120 days, or for 90 days with the following 30 days on control feed. The average daily MOSH intake per animal was calculated based on the MOSH content in the feed and the feed consumption, and was quite consistent over the different exposure durations (Barp et al., 2017a). The nominal MOSH concentrations of 0, 40, 400 and $4000 \mathrm{mg} / \mathrm{kg}$ feed resulted in average daily intake estimates of about $0.016,0.37,3.54$ and $36.36 \mathrm{mg} /$ day, respectively.

\subsubsection{MOSH composition and concentrations in liver}

We have previously reported the data on MOSH concentrations and composition in the spleen, liver (excluding the left liver lobe, which was used for histopathology), adipose tissue and carcass (Barp et al., 2017a). Briefly, there were both a dose-related and a timedependent accumulation of MOSH in the tissues. After 120 days of exposure to control feed and the lowest, middle and highest MOSH dose, the MOSH concentrations in the liver were about 30, 220, 1604 and $5511 \mathrm{mg} / \mathrm{kg}$, respectively. The broad MOSH mixture added to the feed consisted of 31\% n-alkanes and little branched iso-alkanes, 9.9\% multi-branched isoalkanes and 59\% naphthenes. In terms of these broad groups, there was no significant change in composition in the liver. The mass distribution of the MOSH in the liver was more narrow than in the feed and it had a maximum abundance between $n-C_{23}$ and $n-C_{31}$; the most volatile and the highest boiling MOSH were virtually absent. Whereas $n$-alkanes of C16C22 were absent, those between $C_{25}$ and $C_{35}$ were retained (Barp et al., 2017a). 


\subsubsection{Clinical animal data}

No deaths, abnormal behavior or altered physical appearance were observed during the study. Body weight gain (Table 1) and feed intake (data not shown) were not affected by dietary exposure to the broad MOSH mixture, even at the highest dose tested. Whereas the spleen weight was not affected, the weight of the liver was significantly increased in animals exposed to the $4000 \mathrm{mg} / \mathrm{kg}$ dose for 30, 90 and 120 days (Table 1). There were also significant increases in the hepatosomatic index (HSI, i.e. the ratio of liver weight to body weight) in the same groups. In animals exposed to MOSH during 90 days and then fed control diet for 30 days, no such increase was observed, suggesting that the effect on liver weight is reversible.

Table 1: Weight of animals, weight of the organs (liver and spleen), as well as the hepatosomatic index (HSI; liver/body weight ratio) after exposure to the MOSH mixture in feed for 30, 60, 90 and 120 days, or 90 days followed by 30 days on the control diet The results are presented as means from the 5 animals $\pm S D$.

\begin{tabular}{llllll}
$\begin{array}{l}\text { Exposure } \\
\text { period }\end{array}$ & $\begin{array}{l}\text { Dose } \\
(\mathrm{mg} / \mathrm{kg} \\
\text { feed })\end{array}$ & $\begin{array}{l}\text { Body weight } \\
(\mathrm{g})\end{array}$ & $\begin{array}{l}\text { Spleen } \\
(\mathrm{g})\end{array}$ & $\begin{array}{l}\text { Liver } \\
(\mathrm{g})\end{array}$ & HSI \\
(days) & & & & \\
\hline 30 & 0 & $137.0 \pm 5.34$ & $0.44 \pm 0.013$ & $5.69 \pm 0.28$ & $0.0415 \pm 0.0011$ \\
& 40 & $137.2 \pm 8.81$ & $0.45 \pm 0.029$ & $5.73 \pm 0.53$ & $0.0417 \pm 0.0018$ \\
& 400 & $141.4 \pm 8.26$ & $0.48 \pm 0.019$ & $6.27 \pm 0.34$ & $0.0444 \pm 0.0023$ \\
& 4000 & $142.8 \pm 3.96$ & $0.48 \pm 0.014$ & $6.71 \pm 0.25 * *$ & $0.0470 \pm 0.0026^{* *}$ \\
& 0 & $177.6 \pm 5.03$ & $0.54 \pm 0.042$ & $6.57 \pm 0.34$ & $0.0370 \pm 0.0011$ \\
& 40 & $177.2 \pm 6.50$ & $0.55 \pm 0.019$ & $6.23 \pm 0.52$ & $0.0352 \pm 0.0029$ \\
& 400 & $173.0 \pm 11.62$ & $0.54 \pm 0.036$ & $6.20 \pm 0.46$ & $0.0358 \pm 0.0017$ \\
& 4000 & $174.8 \pm 5.63$ & $0.59 \pm 0.041$ & $6.75 \pm 0.35$ & $0.0386 \pm 0.0013$
\end{tabular}




\begin{tabular}{llllll}
90 & 0 & $178.0 \pm 23.56$ & $0.55 \pm 0.053$ & $5.79 \pm 0.79$ & $0.0325 \pm 0.0010$ \\
& 40 & $196.4 \pm 9.56$ & $0.58 \pm 0.040$ & $6.55 \pm 0.25$ & $0.0334 \pm 0.0010$ \\
& 400 & $193.0 \pm 11.60$ & $0.61 \pm 0.054$ & $6.78 \pm 0.47^{*}$ & $0.0351 \pm 0.0014^{*}$ \\
120 & 4000 & $190.6 \pm 9.45$ & $0.62 \pm 0.044$ & $6.86 \pm 0.11^{* *}$ & $0.0361 \pm 0.0022^{* *}$ \\
& 0 & $194.0 \pm 13.73$ & $0.57 \pm 0.043$ & $6.39 \pm 0.81$ & $0.0328 \pm 0.0018$ \\
& 40 & $199.6 \pm 10.99$ & $0.55 \pm 0.042$ & $6.36 \pm 0.42$ & $0.0319 \pm 0.0008$ \\
& 400 & $196.0 \pm 15.35$ & $0.65 \pm 0.111$ & $6.42 \pm 0.65$ & $0.0328 \pm 0.0016$ \\
& 4000 & $206.6 \pm 10.62$ & $0.69 \pm 0.060$ & $7.48 \pm 0.46 *$ & $0.0362 \pm 0.0009 * *$ \\
& 0 & $203.4 \pm 20.71$ & $0.59 \pm 0.072$ & $6.92 \pm 0.77$ & $0.0340 \pm 0.0012$ \\
& 40 & $192.6 \pm 5.13$ & $0.56 \pm 0.036$ & $6.40 \pm 0.43$ & $0.0332 \pm 0.0014$ \\
& 400 & $192.6 \pm 13.54$ & $0.59 \pm 0.052$ & $6.78 \pm 0.79$ & $0.0351 \pm 0.0018$ \\
& 4000 & $188.6 \pm 5.98$ & $0.64 \pm 0.056$ & $6.50 \pm 0.32$ & $0.0345 \pm 0.0012$ \\
\hline
\end{tabular}

${ }^{*}=$ significantly different from control $(p<0.05) ;{ }^{* *}=$ significantly different from control $(p<0.01)$. 


\subsubsection{Histopathological analyses of liver samples}

\section{Granuloma}

There were no significant differences in granuloma density (Fig. 1A) between the groups after 30 and 60 days of exposure. After 90 and 120 days, the density was significantly increased in comparison with the control for the group fed the highest dose $(4000 \mathrm{mg} / \mathrm{kg}$ feed) and did not decrease after a 30 days depuration period. A representative example of liver granuloma is shown in Fig. $2 \mathrm{~A}$. 
A) Granuloma

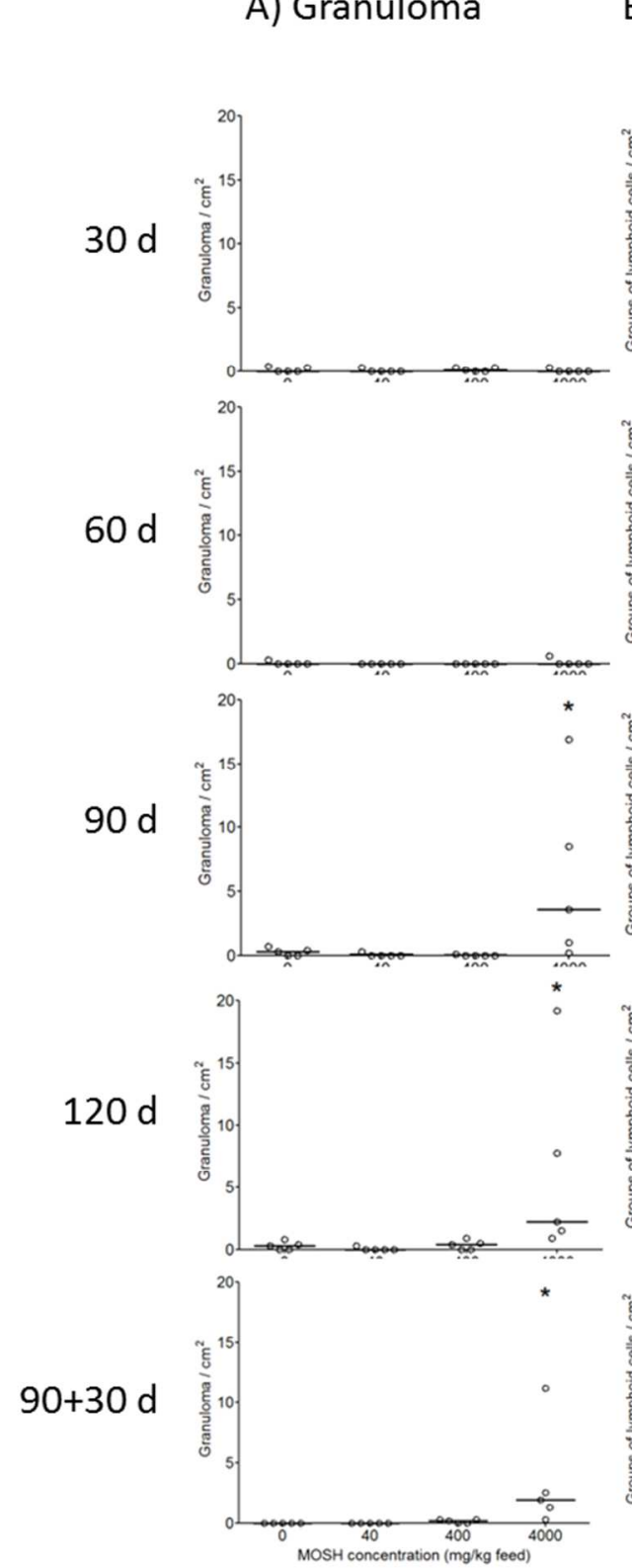

B) Lymphoid clusters portal tracts
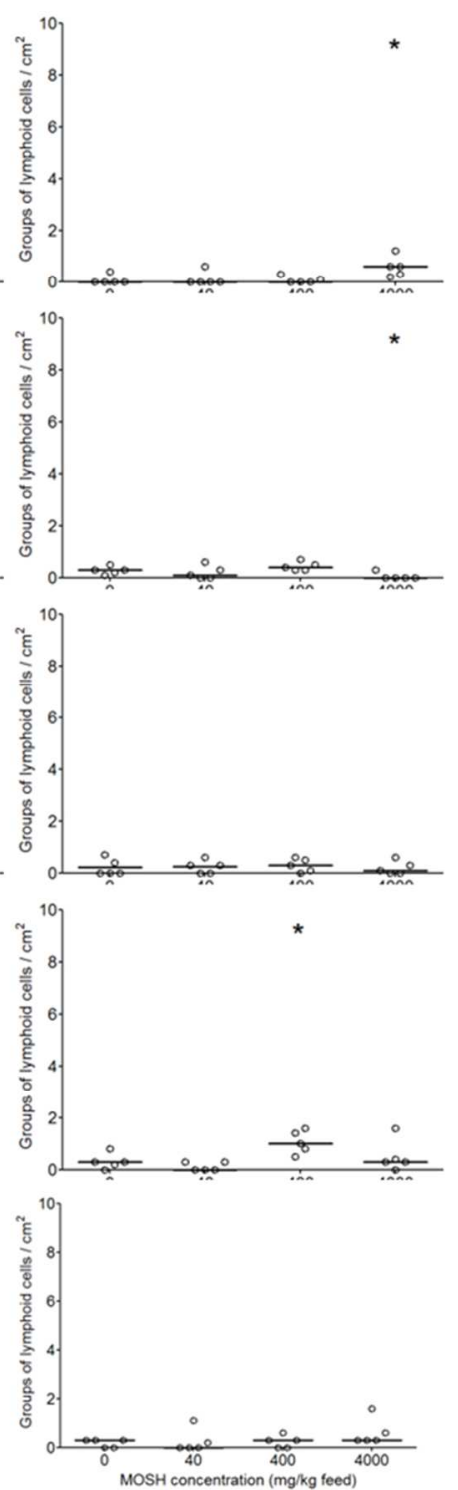

C) Lymphoid clusters parenchyma
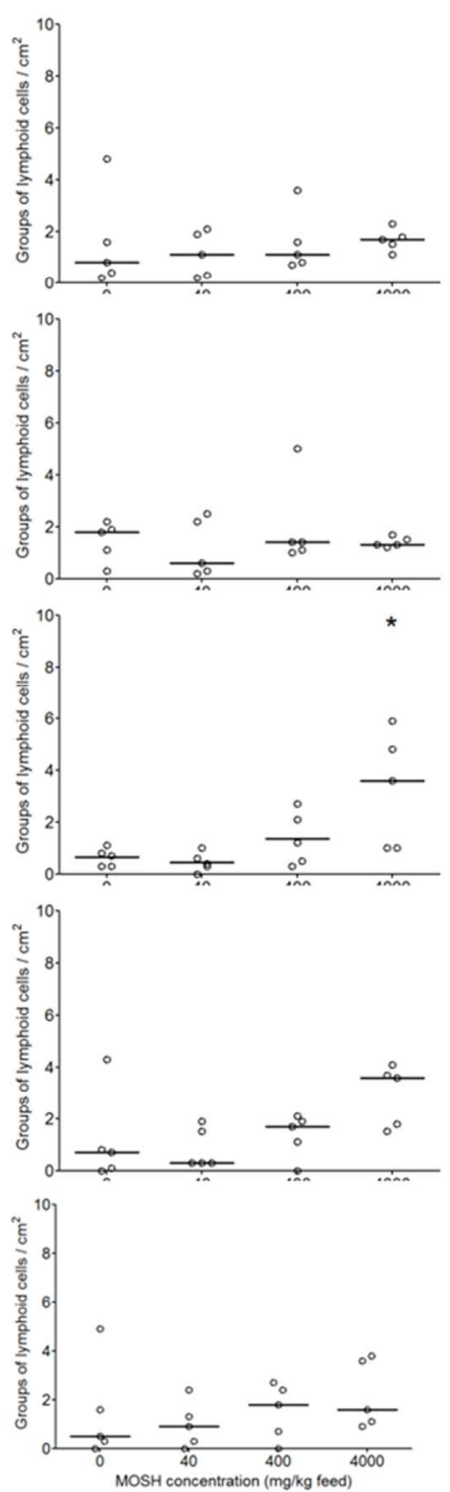

Figure 1. The numbers of granuloma $/ \mathrm{cm}^{2}(A)$, lymphoid cell clusters in the portal tracts $/ \mathrm{cm}^{2}(B)$ and lymphoid cell clusters in the parenchyma/cm ${ }^{2}$ (C) in livers from rats fed for 30, 60, 90 and 120 days with control feed or feed containing MOSH mixture $40 \mathrm{mg} / \mathrm{kg}, 400 \mathrm{mg} / \mathrm{kg}$ and $4000 \mathrm{mg} / \mathrm{kg}$, or for 90 days with the test feed followed by 30 days of control feed $(90+30 d)$. The symbols represent values for the individual animals $(n=5)$ while the lines represent the group median value. Significant differences between treatment and the control groups are denoted by * $(p<0.05)$ 

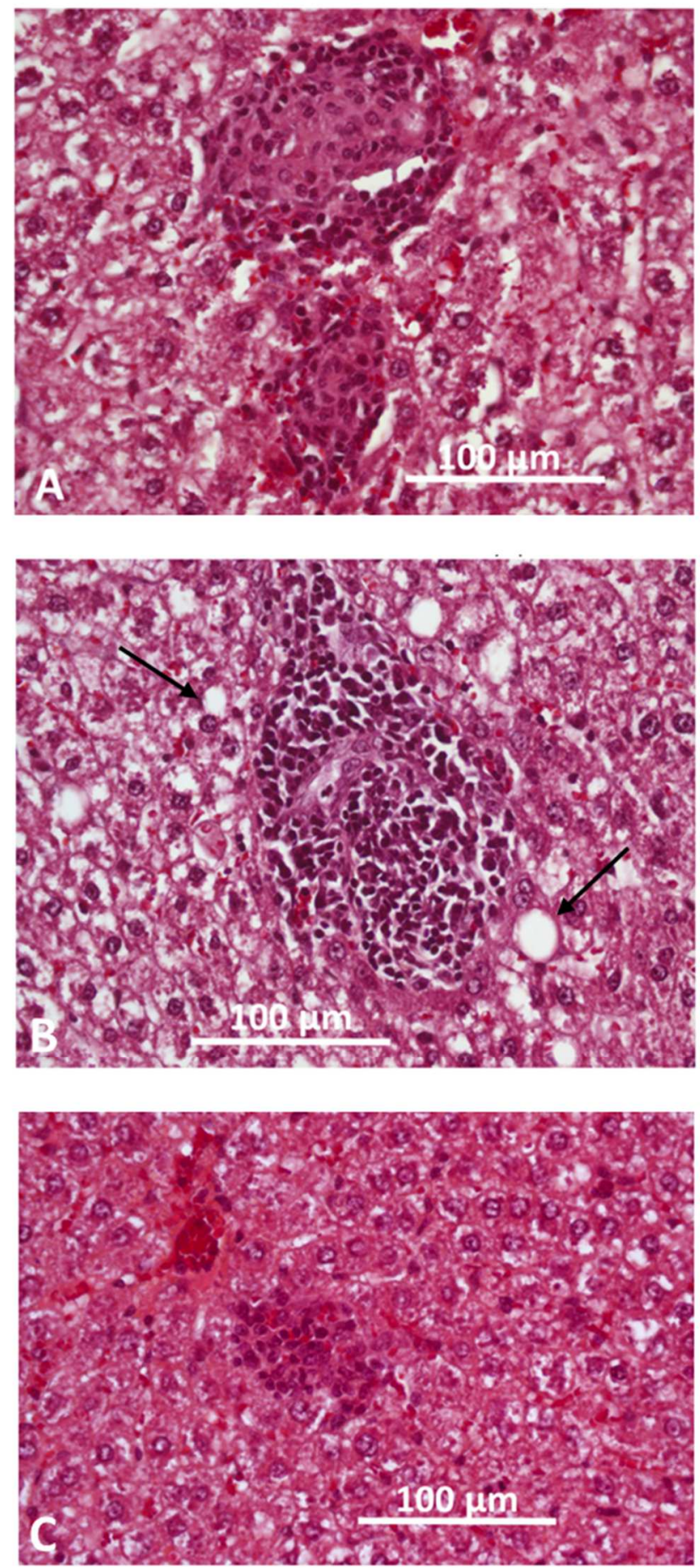

Figure 2: Photos from the histological evaluation of rat livers, showing representative examples of A) a liver specimen with granuloma B) a lymphoid aggregate in a portal tract and C) a lymphoid aggregate in liver parenchyma. The black arrows point to examples of round vacuoles. Magnification indicated by tool bars. 
The relationship between the MOSH accumulated in the liver over 30, 60, 90 and 120 days of exposure and the granuloma density was further examined. MOSH concentrations in liver were measured for four individual animals having received 400 and $4000 \mathrm{mg} / \mathrm{kg} \mathrm{MOSH}$, but only for the pooled livers of three animals exposed to the control feed or the $40 \mathrm{mg} / \mathrm{kg}$ dose (Barp et al., 2017a). There was an apparently exponential relationship between MOSH concentration in the liver and liver granuloma density (Fig. 3).

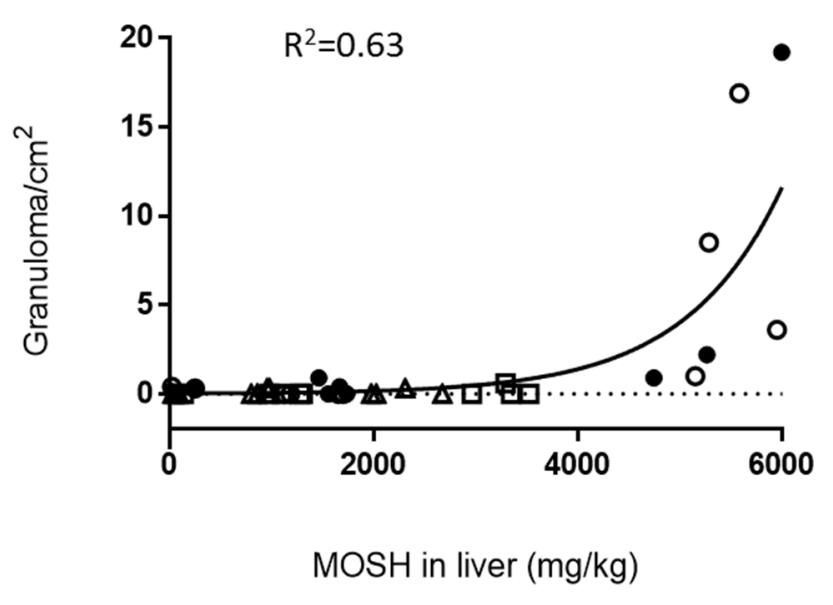

Figure 3: The relation between the measured amount of accumulated MOSH and the respective granuloma density in liver, after $30(\Delta), 60$ (), 90 (०) and 120 (•) days of exposure to the MOSH mixture in feed (experiment 1). The symbols represent levels for individual rats while the line represent an exponential curve fit model, the goodness of fit to the model is expressed as $R^{2}$ (performed in Graphpad Prism7). Up to four individual rats per group per time point were assessed.

\subsubsection{Lymphoid cell clusters in portal tracts}

A representative example of lymphoid cell clusters in the liver portal tracts is shown in Fig. 2B. The numbers of such clusters $/ \mathrm{cm}^{2}$ (Fig. 1B) were low for all animals, but statistically significantly increased at the highest MOSH dose after 30 days of exposure and at the 400 
$\mathrm{mg} / \mathrm{kg}$ feed dose after 120 days, whereas no change was seen for the lowest dose. As there was no clear dose-dependent or time-dependent relationship between MOSH exposure and density of lymphoid clusters in the portal tracts, it is uncertain whether the observed changes were related to the treatment.

\subsubsection{Lymphoid cell clusters in parenchyma}

A representative example of lymphoid cell clusters in the liver parenchyma is shown in Fig. 2C. The numbers of lymphoid cell clusters in the liver parenchyma $/ \mathrm{cm}^{2}$ (Fig. 1C) were in general low, but significantly increased at the highest MOSH dose (4000 mg/kg feed) only, and only after 90 days of exposure. A similar but non-significant tendency of increased numbers at the highest dose was also observed after 120 days of exposure, as well as after 90 days exposure followed by a 30 days period on control feed, since no animals in these groups had zero lymphoid clusters and the group median tended to be higher than for the other groups.

\subsubsection{Liver cell vacuolization}

Round vacuoles in liver cells, as illustrated in Fig. 2B (black arrows), were observed in all livers, even in the controls where they occurred to a slight degree. The degree of vacuolization of the liver cells, indicative of deposited accumulated MOSH material, was semi-quantitatively assessed (see materials and methods section); examples of different vacuolization degrees (slight, moderate and abundant) are presented in Fig. 4 (A, B and C, respectively). The degree of vacuolization varied from no to moderate, but did not differ between the MOSH mixture exposure groups at any exposure duration (data not shown). 

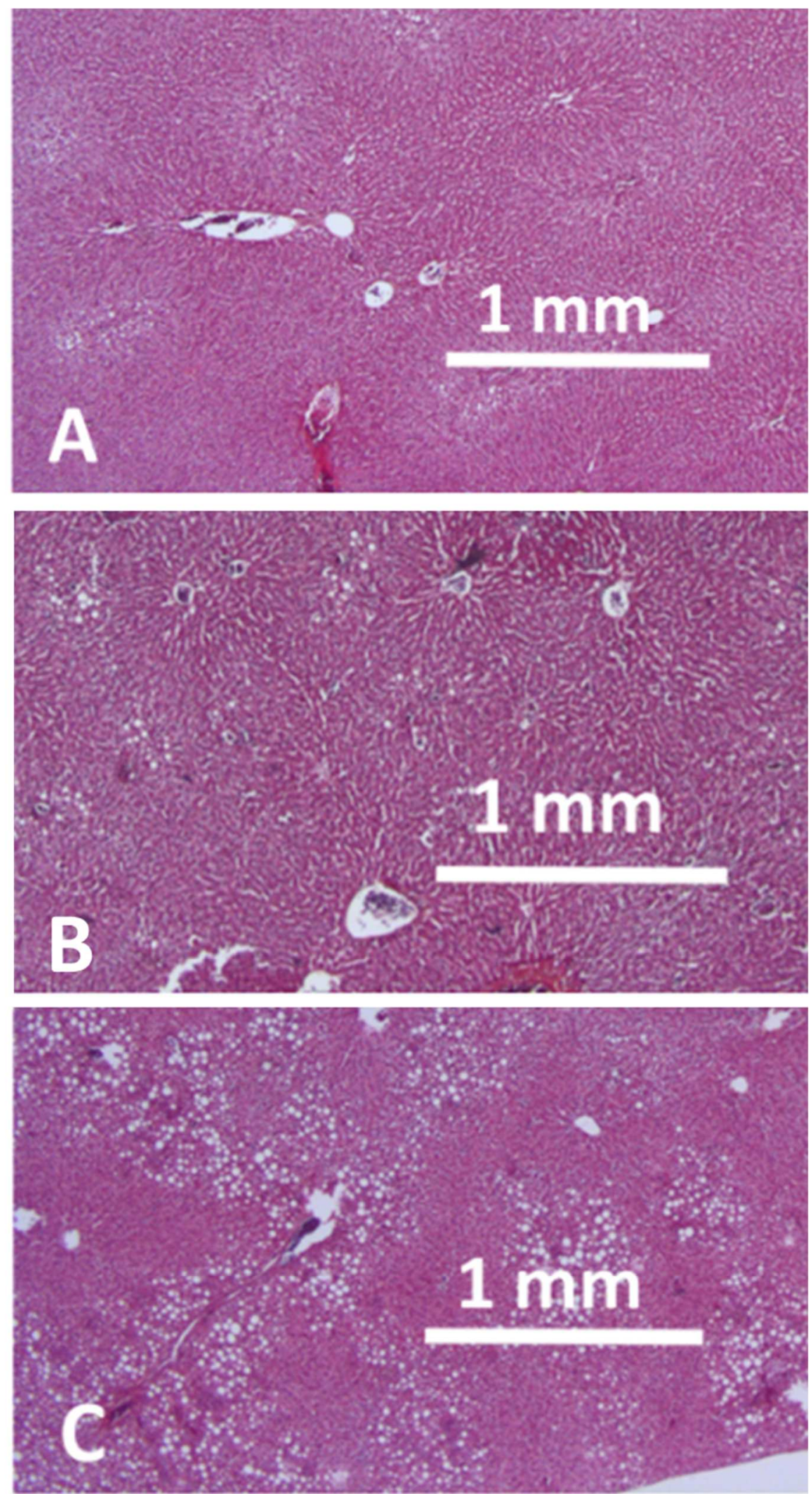

Figure 4: Representative examples of liver sections with vacuolization $A)<5 \%$ (slight); B) $5-15 \%$ (medium) and C) > $15 \%$ (abundant) of the liver cells. Magnification indicated by tool bars. 


\subsection{Experiment 2 - MOSH sub-fractions}

Three dose levels of three different MOSH fractions, S-C25, L-C25 and L-C25W, were administered in feed to groups of rats for 120 days. No differences in feed intake were observed between groups, including controls (data not shown). The dietary concentrations of 400,1000 and $4000 \mathrm{mg} / \mathrm{kg}$ feed corresponded to an average daily MOSH intake over the 120 days of exposure of 3.62, 9.63 and $35.8 \mathrm{mg} /$ day for S-C25, of 3.90, 9.89 and 36.35 $\mathrm{mg} /$ day for L-C25 and 3.44, 8.18 and $34.01 \mathrm{mg} /$ day for L-C25W (for details see Barp et al (2017b)).

\subsubsection{MOSH in the liver}

An extensive characterization of the MOSH concentrations and composition in the spleen, liver, adipose tissue and carcass has been reported in Barp et al. (2017b). In short, for S-C25 exposed animals the MOSH accumulated in the liver had a gas chromatographic retention ranging from $n-C 20$ to $n-C 32$ and were centred on $n-C 24$ (showing a shift to higher mass in comparison with the broad mixture administered in the feed). The signals from n-alkanes had largely disappeared up to C23, but were enriched above C24 and detectable up to about C32. The accumulated MOSH from L-C25 ranged from $n-C 23$ to about $n-C 40$, centered on nC35 (shift to lower mass) and the chromatogram were topped with peaks representing accumulated open-chain iso-alkanes. n-Alkanes were very low, but detectable (while virtually absent in L-C25). The accumulated MOSH from L-C25W resembled those of L-C25, but were in addition enriched with n-alkanes above C25 (Barp et al., 2017b). 


\subsubsection{Clinical data}

No clinical symptoms or signs were observed at any dose level during the course of the study, and there were no statistically significant group differences in body weight (Table 2). No effects on spleen and liver weights were observed in the rats fed the S-C25 fraction, whereas in the groups exposed to L-C25 and L-C25W MOSH fractions, the weights of the liver and the spleen were higher than in the controls, statistically significantly so for the majority of the doses tested (Table 2). Replacement of half of the L-C25 with the wax (LC25W) apparently had stronger impact on spleen and liver weights, indicating that $n$-alkanes above C25 from the wax further increased liver weights compared with L-C25. We further examined whether the increase in liver weight was associated with the measured accumulation of MOSH. In spite of considerable accumulation of MOSH in the liver after SC25 exposure, liver weights were not affected (Fig. 6A). Following L-C25 and L-C25W exposure the increased liver weights were linearly related to accumulated amount of MOSH

(Fig. 6B and C, respectively), but with different slopes; 0.0004 and 0.0003 , respectively. The difference in slope for the L-C25 fraction with and without wax, together with the lack of increase for the S-C25 fraction, illustrates that the increased liver weights were not a direct result of the amount of MOSH accumulated, but was also dependent of the chemical composition of the MOSH. 
Table 2: Weight of animals, weight of the organs (liver and spleen), as well as the hepatosomatic index (HSl; liver/body weight ratio) after exposure to the MOSH fractions S-C25, L-C25 and L-C25W in feed for 120 days. The results are presented as means from 5 animals $\pm S D$.

\begin{tabular}{|c|c|c|c|c|c|}
\hline Groups & $\begin{array}{l}\text { Dose } \\
\text { (mg/kg } \\
\text { feed) }\end{array}$ & $\begin{array}{l}\text { Body weight } \\
\text { (g) }\end{array}$ & $\begin{array}{l}\text { Spleen } \\
\text { (g) }\end{array}$ & $\begin{array}{l}\text { Liver } \\
\text { (g) }\end{array}$ & HSI \\
\hline Control & 0 & $211.3 \pm 6.7$ & $0.62 \pm 0.03$ & $6.76 \pm 0.38$ & $0.0320 \pm 0.0019$ \\
\hline \multirow[t]{3}{*}{ S-C25 } & 400 & $208.8 \pm 14.57$ & $0.63 \pm 0.04$ & $6.82 \pm 0.80$ & $0.0325 \pm 0.0019$ \\
\hline & 1000 & $210.5 \pm 13.20$ & $0.64 \pm 0.03$ & $6.91 \pm 0.90$ & $0.0327 \pm 0.0026$ \\
\hline & 4000 & $205.1 \pm 9.16$ & $0.64 \pm 0.05$ & $6.87 \pm 0.70$ & $0.0335 \pm 0.0025$ \\
\hline \multirow[t]{3}{*}{ L-C25 } & 400 & $213.0 \pm 13.89$ & $0.70 \pm 0.05^{* *}$ & $7.43 \pm 0.49$ & $0.0349 \pm 0.0012^{* *}$ \\
\hline & 1000 & $208.9 \pm 10.71$ & $0.87 \pm 0.15^{* * *}$ & $7.86 \pm 0.60$ & $0.0376 \pm 0.0020 * * *$ \\
\hline & 4000 & $211.3 \pm 13.16$ & $1.17 \pm 0.11 * * *$ & $8.43 \pm 0.70 * *$ & $0.0399 \pm 0.0019 * * *$ \\
\hline \multirow[t]{3}{*}{ L-C25W } & 400 & $203.3 \pm 9.41$ & $1.07 \pm 0.35^{* *}$ & $8.10 \pm 1.14 *$ & $0.0399 \pm 0.0063^{* *}$ \\
\hline & 1000 & $207.3 \pm 9.00$ & $1.22 \pm 0.20 * * *$ & $9.05 \pm 1.66 * * *$ & $0.0437 \pm 0.0082 * *$ \\
\hline & 4000 & $209.8 \pm 4.95$ & $1.25 \pm 0.23^{* * *}$ & $9.09 \pm 1.29 * * *$ & $0.0433 \pm 0.0060 * * *$ \\
\hline
\end{tabular}

\subsubsection{Histopathological analyses of liver samples}

\section{Granuloma}

After 120 days of exposure, the group fed $4000 \mathrm{mg} / \mathrm{kg}$ of the MOSH fraction with carbon numbers below C25 (S-C25) demonstrated significantly increased granuloma density (Fig. 5A). The granuloma density varied by a factor of more than 10 among the individual rats in the highest dose group. A slightly, but not statistically significantly, increased density could also be seen at the second highest dose level of S-C25, $1000 \mathrm{mg} / \mathrm{kg}$. The group exposed to 
the MOSH fraction with carbon numbers above C25 (L-C25) did not show any increase in granuloma formation in any of the three dose groups. When the latter MOSH fraction was mixed 1:1 with wax (L-C25W), feed exposure resulted in a strongly increased granuloma density at all three dose levels, the two highest doses showing a similarly high level of granuloma formation, suggesting that a plateau level was reached.

The relationship between the accumulated MOSH measured in liver and the granuloma density was further investigated by including data from the two animals per group where individual MOSH measurements in liver were performed (Fig. 6D, E, F). Although the data points per group are few, the figures clearly illustrates how the three fractions induced different amounts of total MOSH accumulated in the liver, as well as highly different granuloma densities. While granuloma density followed an apparent dose-dependent response versus the accumulated MOSH in liver after intake of S-C25 (Fig. 6D), the amount of accumulated MOSH in the rats receiving L-C25W was not clearly related to granuloma density within the dose range tested (Fig. 6F). 
A) Granuloma

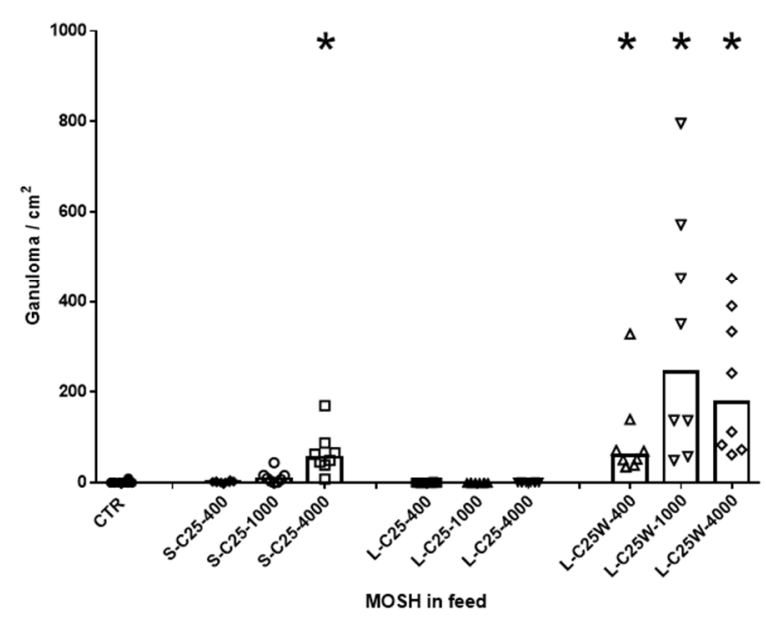

C) Lymphoid clusters in parenchyma

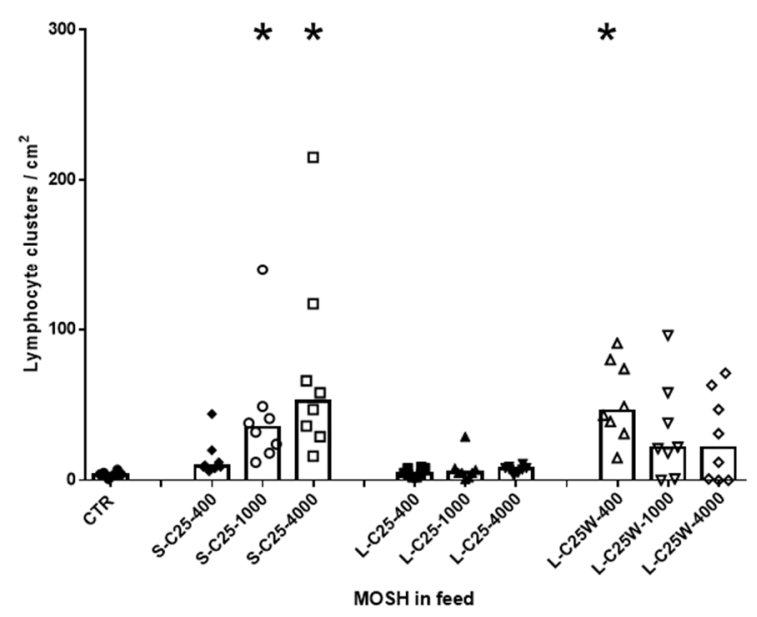

B) Lymphoid clusters in portal tract

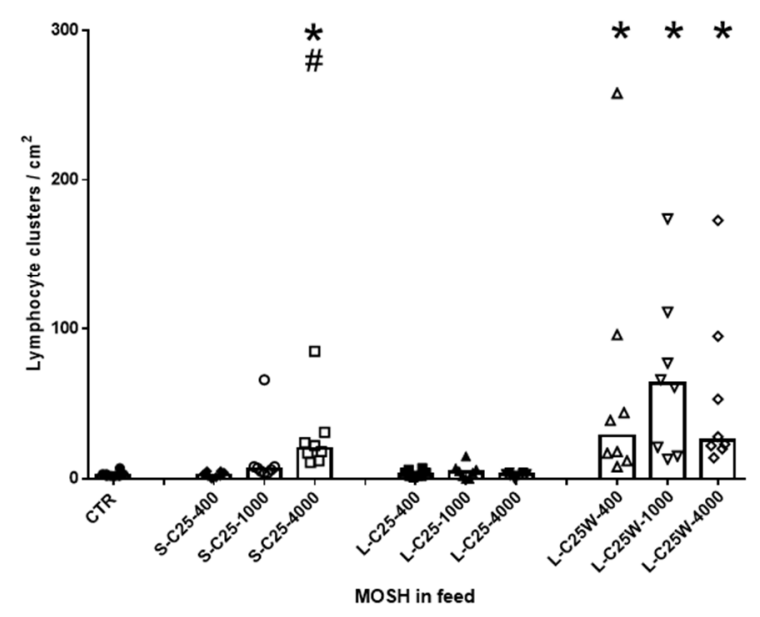

D) Degree of vacuolization

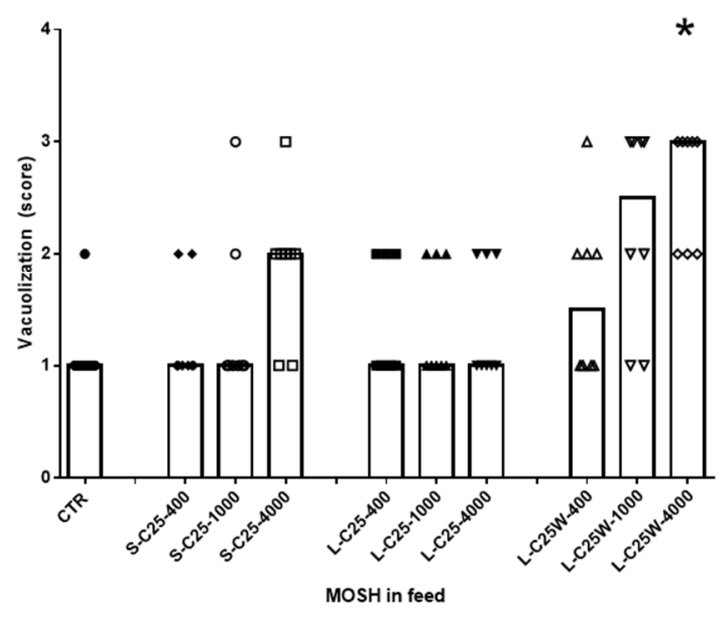

Figure 5: Histopathological evaluation of livers from rats fed for 120 days with control feed or feed containing the MOSH fractions S-C25, L-C25 and L-C25W in doses of 400, 1000 and $4000 \mathrm{mg} / \mathrm{kg}$ feed. A) the numbers of granuloma $/ \mathrm{cm}^{2}$, B) lymphoid cell clusters in the portal tracts $/ \mathrm{cm}^{2}$, C) lymphoid cell clusters in the parenchyma/ $\mathrm{cm}^{2}$, and D) the score representing the degree of vacuolization. Vacuolization grade: 0 - none, 1 -slight (<5\%), 2 - moderate (5-15\%) and 3 -abundant ( $>15 \%)$. The symbols represent values for the individual animals $(n=8)$ while the bars represent the group median value. Significant differences between treatment and the control groups are denoted by * $(p<0.05)$, while \# denotes significant group differences $(p<0.05)$ versus the medium dose of the respective MOSH fraction. 
Liver weights

\section{A) S-C25}

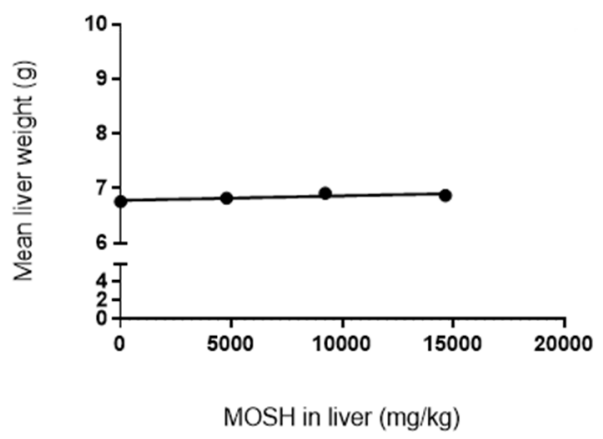

B) L-C25

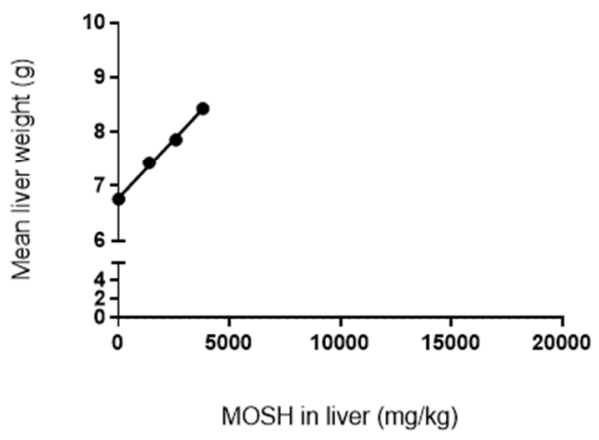

C) L-C25W

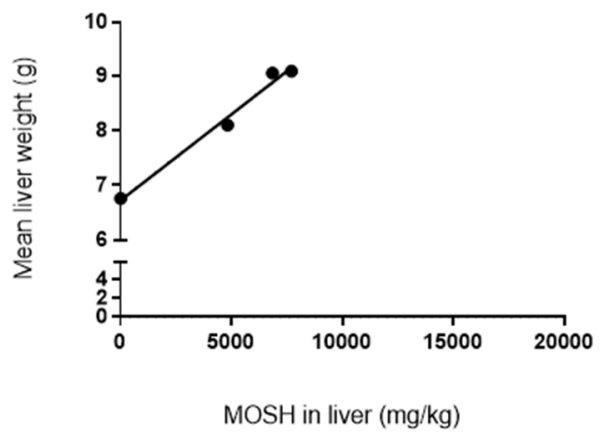

Liver granuloma

D) S-C25

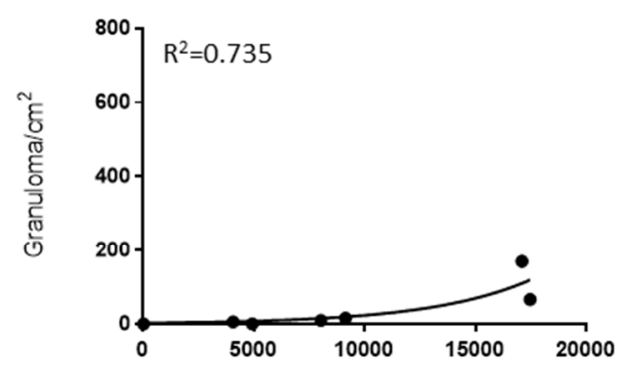

MOSH in liver $(\mathrm{mg} / \mathrm{kg})$

E) L-C25

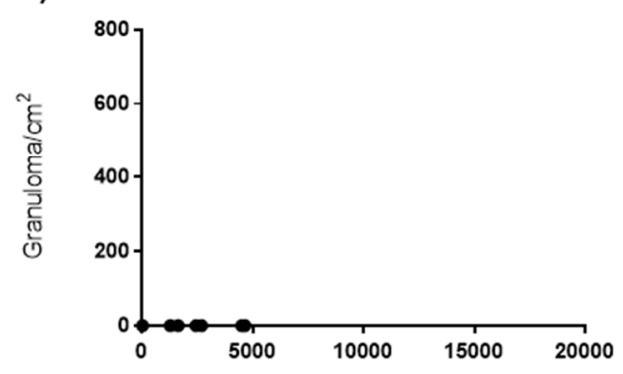

MOSH in liver $(\mathrm{mg} / \mathrm{kg})$

F) L-C25W

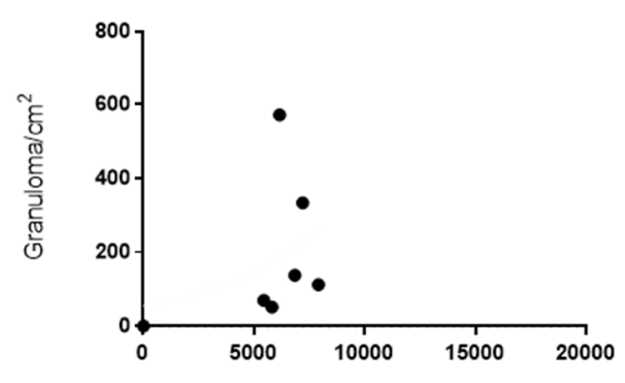

MOSH in liver $(\mathrm{mg} / \mathrm{kg})$

Figure 6: The relation between the measured MOSH accumulated in liver (mg/day, based on results reported in Barp et al., 2017b) and the liver weights (left panel) and granuloma density (right panel) after 120 days of exposure to the three different MOSH fractions S-C25 (A, D), L-C25 (B, E) and L$\mathrm{C25W}$ (C, F). For the left panels, the symbols reflect the group mean values of accumulated MOSH and the corresponding group mean liver weights, and the lines represent a linear fit model. For the right panel, the symbols represent levels for individual rats ( $n=2$ rats with individual MOSH measurements) while the lines represent an exponential curve fit model, the goodness of fit to the model is expressed as $R^{2}$ (performed in Graphpad Prism7). 
Lymphoid cell clusters in portal tract

The numbers of lymphoid cell clusters in the liver portal tracts $/ \mathrm{cm}^{2}$ were not affected by any dose of L-C25 (Fig. 5B). However, all groups receiving the L-C25W fraction and the highest dose of the S-C25 fraction had significantly increased density of lymphoid cell clusters in the portal tracts compared to the control group. The lymphoid cell clusters were also significantly higher in the group fed the highest versus the second highest dose of the S-C25 fractions.

\section{Lymphoid cell clusters in parenchyma}

Similar to the observation in the portal tracts, none of the doses of L-C25 caused any increase in parenchymal lymphoid cell clusters. In the groups fed the S-C25 MOSH fraction there was a dose-dependent increase in the numbers of lymphoid cell clusters in the liver parenchyma $/ \mathrm{cm}^{2}$, statistically significant for the two highest dose levels (Fig. $5 \mathrm{C}$ ). In the rats fed the L-C25W fraction, all dose levels gave a similarly increased density of lymphoid cell clusters.

\section{Liver cell vacuolization and needle shaped clefts}

The semi-quantitatively assessed degree of vacuolization of the liver cells (illustrated in Fig. 4) is presented in Fig. 5D. It was significantly increased for the high dose of L-C25W, scored to be abundant in the majority of the livers in this group. There was also a tendency of increased vacuolization in the groups fed the high dose $(4000 \mathrm{mg} / \mathrm{kg}$ feed) S-C25 and the low and medium dose of L-C25W. 
Besides the round vacuoles in liver cells (as described above), all rats fed L-C25W also displayed oval to needle-shaped clefts in macrophages/epithelioid cells in the granuloma.

Fig. 7 shows examples of these clefts (white arrows and in the frame), as well as of round vacuoles in the liver cells (black arrows). The needle-shaped spaces resemble those left after cholesterol crystals in arteriosclerotic plaques in artery walls, hence we hypothesize that they are clefts after crystalloid MOSH material formed from n-alkanes with high melting points. In rats fed the lowest concentration of L-C25W, there were only few such clefts, whereas in those fed the other MOSH fractions or control feed there were none. The vacuolization in the liver cells as described above, observed in all rat livers, and partly also in some macrophages with a yellow/brown hue, have a round form.

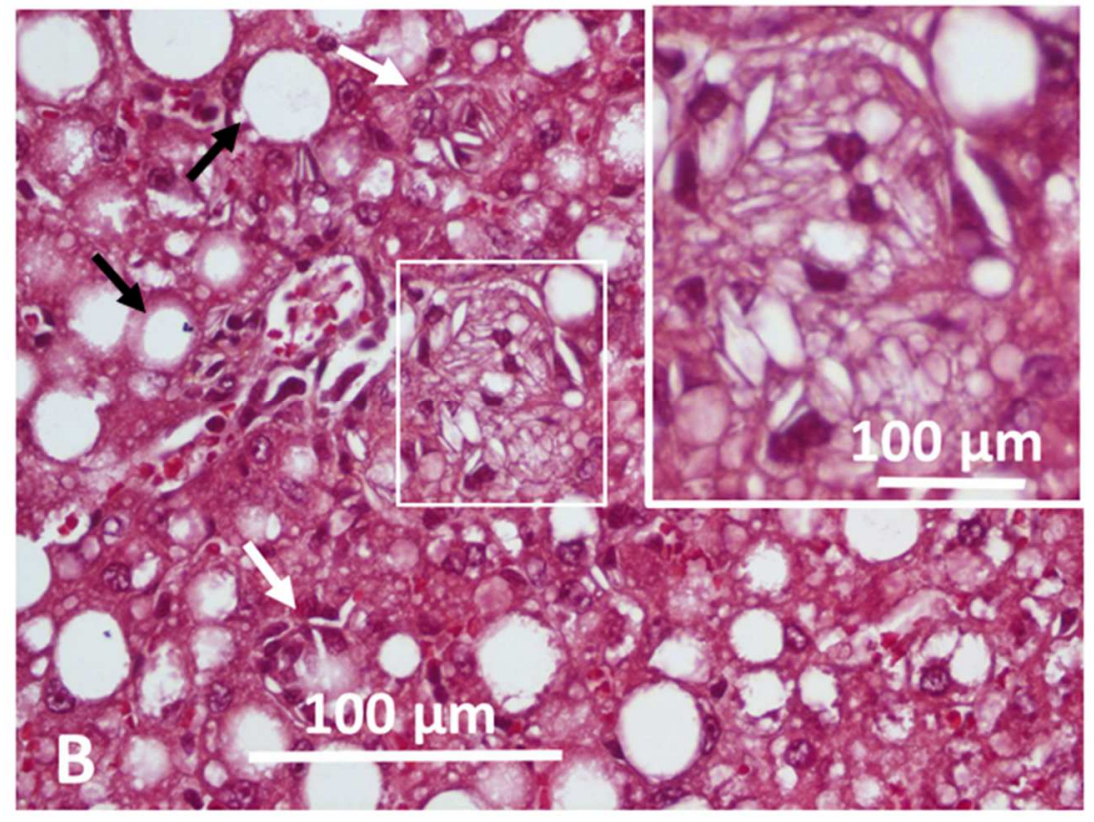

Figure 7: Photo from the histopathological evaluation of rat livers, showing a representative example of a liver specimen with granuloma, round vacuoles in the liver cells (black arrows) and needle-shaped clefts (white arrows and in frame). Magnification indicated by tool bars, the frame is magnified two times in the upper right corner. 


\subsection{Relation between MOSH exposure and hepatic granuloma formation and increase in}

liver weights combining experiment 1 and 2

The second experiment showed that granuloma formation was related to the fraction of the broad MOSH mixture made up of the hydrocarbons going into S-C25 and not those going into L-C25 resulted in granuloma formation. In experiment one using the broad MOSH mixture, $32,8 \%$ of the dose would correspond to the $\mathrm{S}-\mathrm{C} 25$ fraction. Combining the results from experiment one and two, a clear dose response between the estimated intake of S-C25 fraction and granuloma formation at day 120 was observed (Fig. 8A).

The second experiment showed that increased liver weight was related mainly to the fraction of the broad MOSH mixture made up of the hydrocarbons of the L-C25. 32,8\% of the dose of the broad mixture would correspond to the L-C25 fraction. Combining the results from experiment one and two, a clear dose response between the estimated intake of L-C25 fraction and relative increase in liver weight at day 120 was observed (Fig 8B).

A) Granuloma S-C25

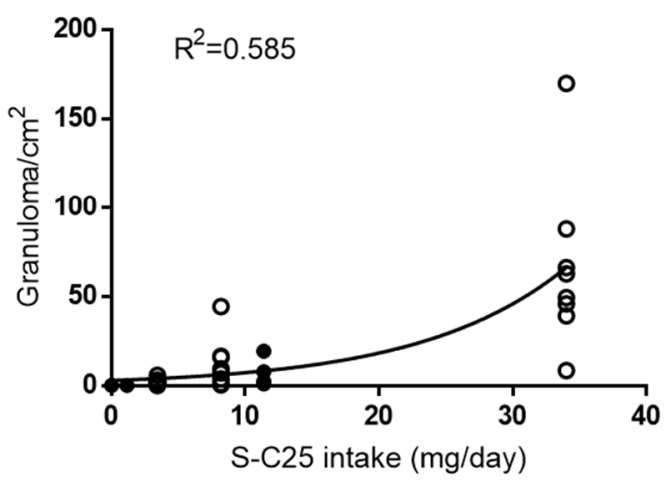

A) Liver weight L-C25

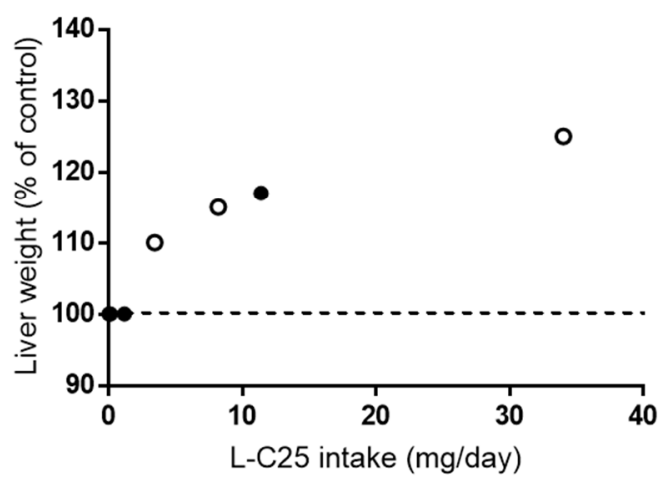

Figure 8: The relation between A) the estimated S-C25 intake ( $\mathrm{mg} /$ day) and the granuloma density and $B$ ) the estimated L-C25 intake ( $\mathrm{mg} /$ day) and liver weight (\% of control, $100 \%$ indicated by a dotted line), after 120 days of exposure in the two experiments. The figure includes data from 
experiment 2 (open symbols) where the animals were exposed to the particular fraction in feed, and from experiment 1 (closed symbols) where the intake of S-C25 and L-C25 was estimated to be 32,8\% of the total MOSH intake after being exposed to the broad MOSH mixture (MOSH intake reported in Barp et al., 2017). The symbols represent levels for individual rats while the lines represent an exponential curve fit model. In $A$, the goodness of fit to the exponential model is expressed as $R^{2}$ (performed in Graphpad Prism7).

\subsection{Immune function analyses}

$\mathrm{KLH}$-specific IgM in serum were detected in all animals, demonstrating that the KLH immunization protocols were successful in eliciting production of KLH-specific IgM. In experiment 1, the IgM concentrations were not significantly different in the four groups of rats (at a dilution of 1:2000; Fig. 9A). In the rats fed the highest MOSH concentration (4000 $\mathrm{mg} / \mathrm{kg}$ feed), however, 4 of 5 animals had low IgM levels in serum, while the fifth animal had a remarkably high response compared to all other animals. The trend of reduced antibody concentrations in the highest exposure group was confirmed by the results from a 1:100 serum dilution (data not shown), whereas most values at that dilution were above the assay detection level. On the other hand, in experiment 2, no significant differences between the groups were observed for KLH-specific IgM antibodies (Fig. 9B, serum dilution of 1:5000). Taken together, no clear immunosuppressive effect was observed in these rats after MOSH exposure, as assessed by primary IgM responses to $\mathrm{KLH}$. 
A) Broad MOSH mixture

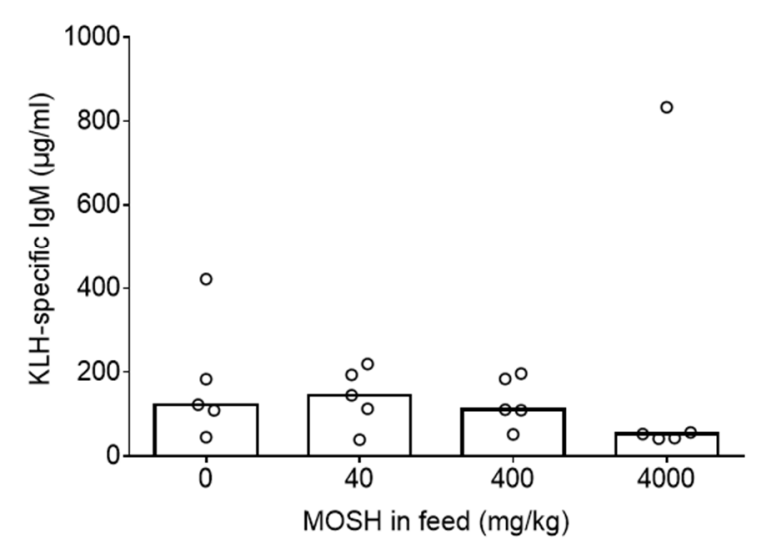

B) Different MOSH fractions

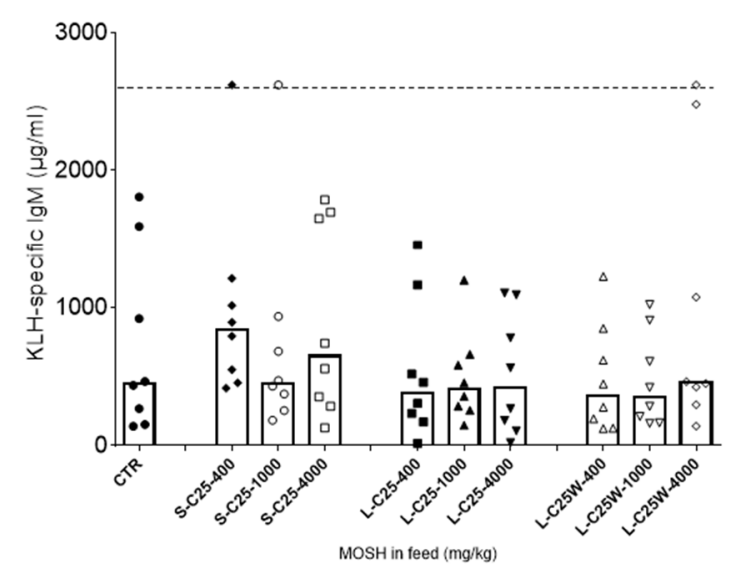

Figure 9: KLH-specific IgM antibodies in serum of rats after 120 days of exposure to MOSH or control feed, 5 days after injection of KLH. A) Animals were exposed to the broad mixture of MOSH at concentrations of 40,400 and $4000 \mathrm{mg} / \mathrm{kg}$ feed ( $n=5)$. Sera were diluted 1:2000. B) Animals were exposed to the three MOSH fractions at concentrations of 400,1000 and $4000 \mathrm{mg} / \mathrm{kg}$ feed (n=8). Sera were diluted 1:5000. The symbols represent the value for the individual animals while the bars represent the group median value. The dotted line indicates the upper detection limit for the ELISA assay. 


\section{Discussion}

The present study provides new information on the importance of the chemical composition of MOSH fractions for effects in rats, and in particular the ability to induce liver granuloma and increased liver weight. In the first experiment, we investigated the effects of a broad MOSH mixture covering the range relevant for human exposure. In the second experiment, we tested two subfractions ( $\mathrm{S}-\mathrm{C} 25$ and L-C25) separated at about $\mathrm{n}-\mathrm{C} 25$ to check the pertinence of the JECFA evaluation in distinguishing the Class I oils from oils of lower molecular mass (Classes II and III), and a low melting point wax (mixed with L-C25 1:1, LC25W) as the wax so far was critical to the toxicological assessment (EFSA 2012).

\subsection{Liver granuloma}

Dietary exposure to a broad MOSH mixture over time induced granuloma formation, associated with influx of lymphoid cells, in the liver. These were not reversible within a 30 days recovery period even if the accumulated MOSH was reduced (Barp et al., 2017a). After 120 days of exposure, $4000 \mathrm{mg} / \mathrm{kg}$ feed of the broad MOSH mixture induced between 2 and 19 granuloma $/ \mathrm{cm}^{2}$, while $4000 \mathrm{mg} / \mathrm{kg}$ of the S-C25 fraction induced between 50 and several hundred granuloma/ $\mathrm{cm}^{2}$ and the L-C25 fraction induced no granuloma. This suggest that some types of hydrocarbons present in the broad mixture and the S-C25 fraction induced the granuloma. The importance of the hydrocarbon composition, rather than the amount of accumulated $\mathrm{MOSH}$, was further strengthened by the observation that about 3.4 to 3.8 times more total MOSH was accumulated in livers after S-C25 exposure than after L-C25 exposure (right panel of figure 6 and Barp et al. 2017b, suppl. material). The highest amount accumulated in the livers in group L-C25 was less than the accumulated amount in the S-C25 group causing increased granuloma density. 
Some of the oils in the broad mixture (i.a. S-C25) were not so well deparaffinated and contained some $n$-alkanes $>\mathrm{C} 25$ (as could be seen in the adipose tissue where $n$-alkanes were enriched; see fig. 2 in Barp et al (2017b). Thus, we combined the data for granuloma formation at 120 days of exposure from rats exposed to the broad mixture and those exposed to S-C25 in a common dose response curve, showing that the results obtained with the broad mixture and the separate fractions were compatible (Fig. 8). This illustrates that for MOSH with similar chemical qualities, the amount of accumulated material was related to the granuloma density. It also indicate that granuloma formation following exposure to the broad MOSH mixture could be explained by the S-C25 fraction.

The very strong granuloma formation after ingestion of the wax-containing L-C25W fraction support previous studies suggesting that fractions containing $n$-alkanes (wax) are very potent in inducing granuloma in the liver of these animals (Scotter et al., 2003; Smith et al., 1996). The ingestion of feed with L-C25W resulted in MOSH accumulation in the liver with enriched $n$-alkanes between n-C25 and n-C36 (visible in GCxGC; Barp et al. 2017b). Whereas L-C25 contained almost no $n$-alkanes and the concentration of $n$-alkanes in the liver was correspondingly low, the S-C25 was not fully deparaffinated and contained a low proportion of $n$-alkanes above C25 ( $n$-alkanes C25-30 amounted to $0.4 \%$ of the total S-C25, equivalent to an exposure of $0.77 \mathrm{mg} /$ day). These $n$-alkanes were in fact enriched in the liver as were the $n$-alkanes of the wax in L-C25W (Barp et al., 2017b). We attempted to estimate the accumulated $n$-alkanes in the liver, but quantification was uncertain and the estimated levels did not show any relationship between granuloma density and accumulated $n$-alkanes (data not shown). Neither the amount of MOSH accumulated after exposure to the wax-enriched fraction L-C25W did relate well with the granuloma density (Fig. 6F). This may be due to 
strong, even maximum, granuloma formation already at the lower concentrations tested. Further, due to high density and partly confluence, it was difficult to differentiate between single granuloma and therefore granuloma density figures were uncertain at the highest densities. Taken together, our observations suggest that other characteristics than the amount of accumulated $n$-alkanes, such as molecular weight and melting point, also are strong determinant of granuloma formation and further studies are needed to resolve these issues.

For the wax-enriched fraction (L-C25W), no clear dose-related response were observed within the present dose range, neither for granuloma formation (two highest doses) nor for the lymphoid clusters (all doses). Neither did we observe any relationship between accumulated MOSH in the liver and granuloma density (Fig. 6C) in rats exposed to L-C25W. In agreement, Griffis et al. (2010) reported that after 90 days of exposure, the severity of hepatic granuloma or lymphocyte infiltrates formation was similar in the groups fed 2000 and $20000 \mathrm{mg} / \mathrm{kg}$ feed low melting point paraffin wax (LMPW). Griffis et al. (2010) also reported that the incidence and severity of the hepatic effects from LMPW increased with time, observed mainly after 60 and 90 days of exposure. The broad mixture used in our study induced liver granuloma first after 90 and 120 days, but the lack of observed effects at 60 days is probably due to the fact that the broad MOSH mixture had a lower potency than LMPW, as it also clearly has a lower potency of granuloma induction than the S-C25 and LC25W fractions. 


\subsection{Lymphoid cell clusters}

Parenchymal and portal lymphoid cell aggregates are commonly observed in the majority of rat livers (McInnes, 2012). This was presently confirmed by the observations of such lymphoid cell clusters after exposure to both control feed and the different concentrations of the broad MOSH mixture. For experiment 2 with the MOSH fractions, however, the pattern was somewhat different; the formation of lymphoid cell aggregates seemed to be increased along with strong granuloma formation. In fact, granuloma formation and the lymphoid cell clusters in the portal tract had a correlation coefficient (Pearson) of 0.650 $(p<0.001 ;$ data not shown). It should be noted that the lymphoid cells reported in this study are groups of cells not associated with the granuloma. The clinical relevance of lymphoid cell clusters in the parenchyma is unknown. Lymphoid cells in the portal tracts, however, are a common finding in human livers and unless they are extensive and part of a hepatitis, they do not seem to be of any clinical relevance in humans. On the other hand, most of the granuloma presently observed had lymphoid cells associated with them. After oral LMPW exposure, Griffis and coworkers reported increased staining for CD3 (T-cells), CD8a (cytotoxic T-cells) and CD4 (T helper cells), as well as a likely origin of non-resident macrophages in the liver granuloma of F-344 rats (Griffis et al., 2010). The presence of lymphoid cell aggregates in the parenchyma in addition to granuloma-associated lymphoid cells in our study thus suggest a chronic inflammatory response in the liver, which may rise a toxicological concern.

\subsection{Mechanisms of granuloma formation}

Granuloma formation in humans has been observed, and can be induced by a number of agents (Ishak and Zimmerman, 1988; Lamps, 2015). Whereas granuloma are immune cell aggregates formed in response to various "chronic" inflammatory stimuli (Herrtwich et al., 
2016), granuloma formation has also been reported as a response to foreign particulate material (Ishak and Zimmerman, 1988; Lamps, 2015). The latter occurs particularly in tissues where such foreign bodies are more common than in liver, like lung or skin (Donaldson et al., 2006). High melting point $n$-alkanes above $\mathrm{n}-\mathrm{C} 25$ showed a steep increase in the liver (Barp et al., 2017b). If crystallization of these occurred, as suggested below, such crystals might have contributed to trigger the granuloma formation. Moreover, the clefts, likely after crystals, were observed only in rats fed the wax-containing MOSH fractions, and far from all granuloma contained such clefts. Accordingly, the results do not indicate that all granuloma were initiated by crystals as foreign bodies, but that also other forms of accumulated MOSH might induce granuloma formation. On the other hand, we speculate that a reason for not seeing needle shaped clefts after $n$-alkane crystals or a clear relationship between accumulated $n$-alkanes and granuloma, could be that particulate material formed from the $n$-alkanes could be a transient phenomenon. It's worth noting that the MOSH material accumulating in human liver, multi-branched or multi-alkylated cycloalkanes, and iso-alkanes (Biedermann et al., 2015), resembles that found in rat liver following L-C25 exposure (Barp et al 2017b), which did not induce liver granuloma.

\subsection{Liver and spleen weights}

In the groups exposed to the L-C25 and L-C25W MOSH fractions, the liver and spleen weights were higher than in the controls, apparently for all dose levels (Table 2). These data complement those demonstrating increase in absolute and relative liver weights after high levels of the broad MOSH mixture (Table 1) and after exposure to diets containing 5000 $\mathrm{mg} / \mathrm{kg}$ or more of food grade white oils and $2000 \mathrm{mg} / \mathrm{kg}$ LMPW (Baldwin et al., 1992; Griffis et al., 2010; Smith et al., 1996). The increase in tissue weight was clearly dependent on the 
chemical characteristics of the MOSH fraction, since no effects were observed in the rats fed the S-C25 fraction and the strength of the weight effect slightly differed for L-C25 and LC25W. In agreement, Griffis and coworkers suggested that the MOSH fraction responsible for these effects mainly corresponded to MOSH ranging from n-C25 to n-C45 (Griffis et al., 2010). In our experiments, the presence of $n$-alkanes from the wax in the L-C25W mixture caused only a slight increase in liver weight above that of L-C25 (Fig. 6B and C). The liver weight increase was not directly related to the accumulated amount of total MOSH in liver, but rather to specific chemical fractions, as the S-C25 fraction had no effect and the L-C25 and L-C25W fractions induced liver weight gains in spite of considerable and moderate MOSH accumulation, respectively (Fig. 6). The fractions accumulating in the liver following LC25 exposure were various multi-branched and long, less branched iso-alkanes and multialkylated cycloalkanes (Barp et al., 2017b). It is noteworthy that similar MOSH fractions have been found in human livers. In 37 liver autopsies, the total MOSH concentrations ranged from 14 to $901 \mathrm{mg} / \mathrm{kg}$ with a mean content of $131 \mathrm{mg} / \mathrm{kg}$ (Barp et al., 2014), i.e. about $10 \%$ of that observed after $400 \mathrm{mg} / \mathrm{kg} \mathrm{L-C25}$ in the current experiment (Fig. 6B and Barp et al. 2017b). Increased tissue weights can have multiple causes, and further histological or functional analyses is needed to determine its clinical relevance (Hall et al., 2012). We observed no histopathological changes except for granuloma formation and lymphoid cell clusters, and these endpoints were not related to the increased liver weights (Fig. 6). It is worth noticing that the liver weight effects, in contrast to the granuloma effects, were reversible, as the weights were similar to the control group after 30 days on control feed subsequent to 90 days of feed containing the broad MOSH mixture (Tab.1, Fig 1). Although it is likely that the weight increase was an adaptive response, in humans, the exposure is continuous and an increase in liver weight is likely to be permanent. The clinical significance 
of increased liver and spleen weights, which might occur in humans that are chronically exposed to MOSH, should be further investigated.

MOSH concentrations reported in human spleens (Barp et al., 2014) are in the same range as those caused by the lowest dose of L-C25 in the present study, a dose which significantly increased the spleen weight. It is therefore possible that MOSH exposure to humans might cause an increase in spleen weight, but the clinical significance of increased spleen weights is uncertain. As the present study was designed to fully assess MOSH accumulation in spleen, no tissue was left for histopathology (Barp et al., 2017a; Barp et al., 2017b). If the increased spleen weight in our experiment was caused by mechanisms strongly influencing spleen cell function, one may expect that this would influence the production of KLH-specific IgM antibody levels, although this endpoint is only reflecting one arm of immune function. The observation that IgM levels were not affected even in the groups with the most pronounced increases in spleen weight do not support this notion.

\subsection{Liver vacuoles}

The degree of vacuolization in the livers was determined semi-quantitatively and was related to the amount of MOSH accumulated in the livers, but not to the increased liver weights in the second experiment (Fig. 5D). Such round shaped vacuoles were present also in nonexposed rats, but were somewhat increased even in rats receiving L-C25, the material that apparently was less accumulated. The presence of round vacuoles is similar to lipid droplets that is usually seen in fatty livers both in humans and in rats (Fleming et al., 1998). Here, however, round vacuoles is likely to be spaces left following deposition of mineral oil mixed with lipids as mineral oil is strongly lipophilic and are likely to dissolve in lipids in 
membranes. The accumulation of such droplets might be a result of non-degradable MOSH material in the droplets causing a decrease in turnover by autophagy (Martinez-Lopez and Singh, 2015). In addition, the rats fed L-C25W, but none of the other MOSH fractions, displayed oval to needle-shaped "clefts" or "spaces" in macrophages/epitheloid cells in granuloma (Fig. 7). We assume that these are spaces after crystalloid material. They resemble what can be seen in foamy Kupffer cells in livers of acid lipase deficiency rats (Wolman's disease model rats) (Kuriwaki and Yoshida, 1999) and in atherosclerotic plaques in arteries, where cholesterol is washed out during processing of the tissue.

Chromatographic analysis (Barp et al., 2017b) of livers from the same animals showed that $n$-alkanes in liver were not degraded above $\mathrm{C} 25$ (melting point $53^{\circ} \mathrm{C}$ ) and even enriched, which might be explained by crystallization. This high melting wax material was apparently well absorbed probably because it was dissolved in the oily L-C25 MOSH fraction.

\subsection{Immunotoxicity}

Studies of immunotoxic consequences of oral exposure to MOSH has been requested because the significance of influx of lymphocytes in the liver is unclear. To investigate effects of environmental exposures on the immune system, functional endpoints are recommended (S8 Immunotoxicity studies for Human Pharmaceuticals; (FDA, 2006) and Health Effects Test Guidelines OPPTS 870.7800 Immunotoxicity; (EPA, 1998)). Therefore, we measured KLHspecific IgM in serum after KLH immunization, which has been recommended as a useful marker of immunosuppressive or -stimulating effects in the OECD guideline 407 Repeated dose 28-day oral study in rodents (OECD, 2008). Overall, no significant effects on the KLHspecific IgM concentrations in serum were observed after 120 days of exposure to the three doses of the broad MOSH mixture or to the three MOSH fractions, S-C25, L-C25 and L-C25W. 
Due to the complexity of the immune system, we can obviously only conclude on a small part of immune function, i.e. IgM production by B cells. However, in agreement, the broad MOSH mixture did not induce any detectable immune modulation, including autoimmune mechanisms, after 90 days of dietary exposure in Dark Agouti rats (Andreassen et al., 2017). Only two previous experiments measuring antigen-specific antibodies after MOSH exposure are known, indicating no or decreased levels of antigen-specific antibody production in Fischer 344 rats (but not in Sprague Dawley) (ImmunoTox, 2001). The relation between IgM responses and the inflammatory responses in the liver granuloma is not known nor necessarily expected, but the present lack of immunosuppression or stimulation (determined as antigen-specific IgM antibody production after antigen injection) in the animals showing strong inflammatory granuloma formation in the liver, indicate that these are independent mechanisms.

\subsection{Animal model}

We used female Fischer 344 rats in this study because in previous studies this strain and sex seemed to be the most sensitive one with regard to hepatic granuloma formation following oral MOSH exposure (Griffis et al., 2010). Lack of hepatic granuloma formation, and also weaker MOSH accumulation in tissues, have been reported in other rat strains (Firriolo et al., 1995; Griffis et al., 2010). The inherent inter-strain and inter-species differences in susceptibility have been attributed to the higher absorption and retention and possibly to a generally exacerbated immune mediated response occurring in the Fisher 433 rats (Griffis et al., 2010; Miller et al., 1996). In humans, MOSH accumulation in tissues, including liver, have recently been thoroughly examined (Barp et al., 2014; Biedermann et al., 2015). 


\section{Conclusion}

Dietary exposure to a broad MOSH mixture, covering the chemical composition relevant for human exposure, increased liver and spleen weights, as well as vacuolization and granuloma formation associated with lymphoid cell clusters in the liver. The effects varied strongly between the various MOSH fractions/chemical composition. The increased liver and spleen weights were mainly related to accumulated iso-alkanes and substituted cycloalkanes, but also wax n-alkanes. Induction of liver granuloma appeared to be related to n-alkanes $>$ C25 and not to the amount of MOSH accumulated in the liver. MOSH levels and compositions appear to be similar to those observed in humans. Dietary MOSH exposure, and/or inflammatory granuloma formation in the liver and increased spleen weight, was not associated with suppressed or stimulated immune responses to an injected antigen.

\section{Acknowledgments}

This research was part of a grant project co-funded by the European Food Safety Authority (EFSA), grant agreement GP/EFSA/BIOCONTAM/2013/01 ("Bioaccumulation and toxicity of mineral oil hydrocarbons in rats - specificity of different subclasses of a broad mixture relevant for human dietary exposures"). The findings, conclusions, and opinions expressed in this scientific manuscript reflect only the view of the authors and not the official position of the European Food Safety Authority.

The authors would like to acknowledge Florence Blas-y-Estrada, Perrine Aubry and Xavier Blanc (INRA) for excellent technical assistance with the animal experiments and feed preparation, Johanna ML Eidahl, MSc, Oslo University Hospital, for sectioning and staining of 
the liver specimens, and Else-Carin Groeng at the Norwegian Institute of Public Health for performing the KLH-ELISAs. 


\section{References}

Andreassen M, Hjertholm H, Cravedi JP, Grob K, Alexander J, Nygaard UC. Effect of dietary pristane and other saturated mineral oils (MOSH) on autoimmune arthritis in rats. Toxicol Rep 2017; 4: 104-112.

Baldwin MK, Berry PH, Esdaile DJ, Linnett SL, Martin JG, Peristianis GC, et al. Feeding studies in rats with mineral hydrocarbon food grade white oils. Toxicol Pathol 1992; 20: 426-35.

Barp L, Biedermann M, Grob K, Blas YEF, Nygaard UC, Alexander J, et al. Accumulation of mineral oil saturated hydrocarbons (MOSH) in female Fischer 344 rats: Comparison with human data and consequences for risk assessment. Sci Total Environ 2017a; 575: 15.

Barp L, Biedermann M, Grob K, Blas YEF, Nygaard UC, Alexander J, et al. Mineral oil saturated hydrocarbons (MOSH) in female Fischer 344 rats; accumulation of wax components; implications for risk assessment. Sci Total Environ 2017b; 583: 319-333.

Barp L, Kornauth C, Wuerger T, Rudas M, Biedermann M, Reiner A, et al. Mineral oil in human tissues, Part I: concentrations and molecular mass distributions. Food Chem Toxicol 2014; 72: 312-21.

Biedermann M, Barp L, Kornauth C, Wurger T, Rudas M, Reiner A, et al. Mineral oil in human tissues, Part II: Characterization of the accumulated hydrocarbons by comprehensive twodimensional gas chromatography. Sci Total Environ 2015; 506-507: 644-55.

Donaldson K, Aitken R, Tran L, Stone V, Duffin R, Forrest G, et al. Carbon nanotubes: a review of their properties in relation to pulmonary toxicology and workplace safety. Toxicol Sci 2006; 92: 522.

EFSA. Scientific Opinion on the use of high viscosity white mineral oils as a food additive. EFSA Journal 2009; 7.

EFSA. Scientific Opinion on Mineral Oil Hydrocarbons in Food, EFSA Panel on Contaminants in the Food Chain (CONTAM). EFSA Journal 2012; 10: 185.

EPA. Health Effects Test Guidelines OPPTS 870.7800 Immunotoxicity. U. S. Environmental Protection Agency, 1998. 
FDA. ICH S8 Immunotoxicity Studies for Human Pharmaceuticals In: Administration USDoHaHSFaD, editor, Rockcille, MD, 2006, pp. 9.

Firriolo JM, Morris CF, Trimmer GW, Twitty LD, Smith JH, Freeman JJ. Comparative 90-day feeding study with low-viscosity white mineral oil in Fischer-344 and Sprague-Dawley-derived CRL:CD rats. Toxicol Pathol 1995; 23: 26-33.

Fleming KA, Zimmerman H, Shubik P. Granulomas in the livers of humans and Fischer rats associated with the ingestion of mineral hydrocarbons: a comparison. Regul Toxicol Pharmacol 1998; 27: $75-81$.

Griffis LC, Twerdok LE, Francke-Carroll S, Biles RW, Schroeder RE, Bolte H, et al. Comparative 90-day dietary study of paraffin wax in Fischer-344 and Sprague-Dawley rats. Food Chem Toxicol 2010; 48: 363-72.

Hall AP, Elcombe CR, Foster JR, Harada T, Kaufmann W, Knippel A, et al. Liver hypertrophy: a review of adaptive (adverse and non-adverse) changes--conclusions from the 3rd International ESTP Expert Workshop. Toxicol Pathol 2012; 40: 971-94.

Herrtwich L, Nanda I, Evangelou K, Nikolova T, Horn V, Sagar, et al. DNA Damage Signaling Instructs Polyploid Macrophage Fate in Granulomas. Cell 2016; 167: 1264-1280.e18.

ImmunoTox. Effects of dietary mineral hydrocarbons on T cell dependent antibody production in two strains of rats. . Unpunlished study ITI 1298 prepared for American Petroleum Institute, Washington DC, as descroned nu WHO/IPCS, 20032001.

Ishak KG, Zimmerman HJ. Drug-induced and toxic granulomatous hepatitis. Baillieres Clin Gastroenterol 1988; 2: 463-80.

JECFA. 59th report, 11-20. WHO Technical Report Series. 913. Joint FAO/WHO Expert Committee on Food Additives. , 2002.

Kuriwaki K, Yoshida H. Morphological characteristics of lipid accumulation in liver-constituting cells of acid lipase deficiency rats (Wolman's disease model rats). Pathol Int 1999; 49: 291-7. 
Lamps LW. Hepatic Granulomas: A Review With Emphasis on Infectious Causes. Arch Pathol Lab Med 2015; 139: 867-75.

Martinez-Lopez N, Singh R. Autophagy and Lipid Droplets in the Liver. Annu Rev Nutr 2015; 35: 215-

37.

McInnes EF. Background lesions in laboratory animals. A Color Atlas. Elsevier, 2012, pp. 26.

Miller MJ, Lonardo EC, Greer RD, Bevan C, Edwards DA, Smith JH, et al. Variable responses of species and strains to white mineral oils and paraffin waxes. Regul Toxicol Pharmacol 1996; 23: 5568.

OECD. OECD guideline 407. Repeated Dose 28-Day Oral Toxicity Study in Rodents In: OECD, editor. OECD GUIDELINES FOR THE TESTING OF CHEMICALS, 2008.

Reeves PG, Nielsen FH, Fahey GC, Jr. AIN-93 purified diets for laboratory rodents: final report of the American Institute of Nutrition ad hoc writing committee on the reformulation of the AIN76 A rodent diet. J Nutr 1993; 123: 1939-51.

Scotter MJ, Castle L, Massey RC, Brantom PG, Cunninghame ME. A study of the toxicity of five mineral hydrocarbon waxes and oils in the F344 rat, with histological examination and tissuespecific chemical characterisation of accumulated hydrocarbon material. Food Chem Toxicol 2003; 41: 489-521.

Smith JH, Mallett AK, Priston RA, Brantom PG, Worrell NR, Sexsmith C, et al. Ninety-day feeding study in Fischer-344 rats of highly refined petroleum-derived food-grade white oils and waxes. Toxicol Pathol 1996; 24: 214-30.

Vandebriel RJ, Tonk EC, de la Fonteyne-Blankestijn LJ, Gremmer ER, Verharen HW, van der Ven LT, et al. Immunotoxicity of silver nanoparticles in an intravenous 28-day repeated-dose toxicity study in rats. Part Fibre Toxicol 2014; 11: 21. 